\title{
The Effect of Hanging Device on the Vehicle Natural Frequency
}

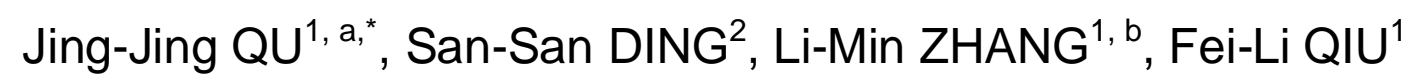

${ }^{1}$ State Key Laboratory of Traction Power, Southwest Jiaotong University, Chengdu, China 610031

${ }^{2}$ CSR Sifang Co.Ltd, Qingdao Shandong 266000, China

a675876357@qq.com, bzhang-Im01@163.com

${ }^{*}$ Corresponding author

Keywords: Vibration and Wave, Natural Frequency, Traction Transformer, Hanging Stiffness.

\begin{abstract}
When the vehicle is running, there is resonance phenomenon if the frequency of excitation is equal to the natural frequency of vehicle. But it can change the frequency of the new system when a traction transformer is hung under the car-body. In a certain extent, it can avoid the resonance. Meanwhile, the spring hanging stiffness of traction transformer affects the natural frequency of the system: the natural frequency of the system increases when hanging stiffness increases. Thereby, hanging transformer and changing the hanging stiffness of traction transformer can change the natural frequency of the system, finally avoid resonance.
\end{abstract}

\section{Introduction}

Resonance is a phenomenon that at a particular frequency, the physical system vibrates with maximum amplitude. When it refers to railway vehicles, if the vehicle is running, it is possible that resonance cause the vehicle to have a greater deformation, or affect the normal operation of the devices, even lead the vehicle to be out of control, resulting in immeasurable serious consequences. However, a running vehicle will be motivated by excitations with various frequencies inevitably. When the frequency of a certain excitation is closed to even equals the natural frequency of the vehicle, it will cause resonance. To prevent this phenomenon, the natural frequency of the system can be changed via hanging transformer and changing hanging stiffness to make the frequency of excitation is unequal to the natural frequency of the new system.

\section{Theoretical Derivation}

When the traction transformer is not hung under the car-body, there is a natural frequency $\omega$.

Assume the car-body for a uniform simply supported beam with mass $M$, length $L$, bending stiffness $E I$ [1], where $E$ is the elastic modulus of the material, $I$ is the moment of inertia of beam section to bypass the geometric center of the beam. When the beam does bending vibration, the displacement of center line at any time is $y(x, t)$. Beam is placed fixed. Structure diagram is shown in Fig.1.

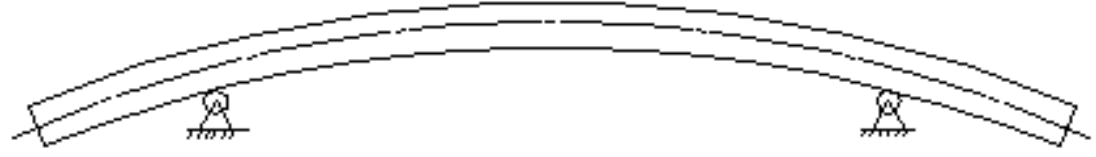

Fig. 1 Structure Diagram without Traction Transformer

The motion equation of freedom vibration system is [2]:

$$
\frac{\partial^{2}}{\partial x^{2}}\left[E I \frac{\partial^{2} y(x, t)}{\partial x^{2}}\right]+M \frac{\partial^{2} y(x, t)}{\partial t^{2}}=0 \quad(0 \leq x \leq L)
$$

It is obtained by separation of variables: 


$$
\frac{\mathrm{d}}{\mathrm{d} x^{2}}\left[E I \frac{\mathrm{d}^{2} Y(x)}{\mathrm{d} x^{2}}\right]=\omega^{2} M Y(x) \quad(0 \leq x \leq L)
$$

That is:

$$
\frac{\mathrm{d}^{4} Y(x)}{\mathrm{d} x^{4}}-\beta^{4} Y(x)=0(0 \leq x \leq L) \quad \beta^{4}=\frac{\omega^{2} M}{E I}
$$

Its general solution is:

$$
Y(x)=C_{1} \sin \beta x+C_{2} \cos \beta x+C_{3} \sinh \beta x+C_{4} \cosh \beta x
$$

By boundary conditions $\left\{\begin{array}{l}Y(0)=0,\left.\frac{\mathrm{d}^{2} Y(x)}{\mathrm{d} x^{2}}\right|_{x=0}=0 \\ Y(L)=0,\left.\frac{\mathrm{d}^{2} Y(x)}{\mathrm{d} x^{2}}\right|_{x=L}=0\end{array}\right.$, with Eq.4, the frequency $\omega$ can be obtained as:

$$
\omega=(r \pi)^{2} \sqrt{\frac{E I}{M L^{4}}} \quad(\mathrm{r}=1,2,3, \cdots)
$$

The first natural frequency is calculated with $r=1$.

$$
\omega=\frac{\pi^{2}}{L^{2}} \sqrt{\frac{E I}{M}}
$$

When the traction transformer is hung under the car-body, there is another natural frequency $\omega_{1}$ of the car-body .Structure diagram is shown in Fig.2.

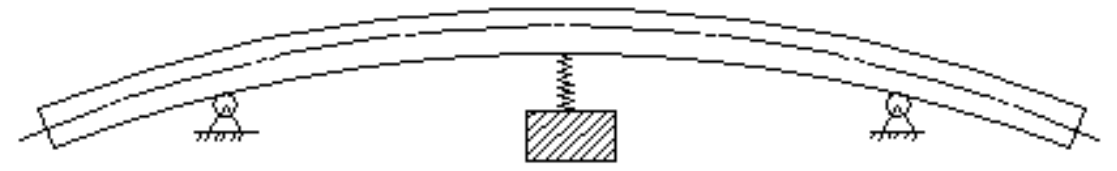

Fig. 2 Structure Diagram with Traction Transformer

The car-body structure is equivalent to the simply supported beam of free vibration, traction transformer is regarded as a block with $m$, and hanging stiffness is $k_{1}$, excitation is $\mathrm{Q}$, then the vibration differential equation for the device is [3]:

$$
m \ddot{y}+k_{1}\left(y-y_{l / 2}\right)=Q(t)
$$

Where, $y$ is horizontal displacement of traction transformer, $y_{l / 2}$ is horizontal displacement of suspension point.

The vibration differential equation of beam is [4]:

$$
E I \frac{\partial^{4} y(x . t)}{\partial x^{4}}+M \frac{\partial^{2} y(x . t)}{\partial t^{2}}+k y(x, t)=0
$$

It is obtained by separation of variables:

$$
y(x, t)=\sum_{j=1}^{\infty} y_{j}(x) \sin \omega_{j} t
$$


It can be got by taking Eq.8 into Eq.7:

$$
E I \frac{\mathrm{d}^{4} y_{j}(x)}{\mathrm{d} x^{4}}+M \omega_{j}^{2} y_{j}(x)+k y_{j}(x)=0
$$

With Eq.3, 4, 9, the natural frequency of car-body can be obtained as:

$$
\omega_{j}=(r \pi)^{2} \sqrt{\frac{E I}{M L^{4}}+\frac{k_{1}}{M L^{4}}} \quad(r=1,2,3, \cdots)
$$

The first natural frequency is calculated with $r=1$.

$$
\omega_{1}=\frac{\pi^{2}}{L^{2}} \sqrt{\frac{E I}{M}+\frac{k_{1}}{M}}
$$

It can be seen that when the block is hung, the natural frequency of the car-body will increase.

To a vehicle without hung traction transformer, its natural frequency is $\omega$. when the vehicle is running, it may be motivated by various frequencies of excitations, when the excitation frequency equals the natural frequency of the vehicle, resonance happens. To avoid this phenomenon, the related devices can be hung at appropriate positions under the car-body. Meanwhile, by the formula Eq11, when the other parameters of the vehicle and traction transformer are unchanged, hanging stiffness between the car-body and traction transformer is the only factor that affects the natural frequency.

\section{Simulation}

The car-body structure is mainly composed of four parts: side walls, roof, headwalls and other components. The finite element model is constructed according to the actual structure characteristics of the car-body. Based on the three-dimensional model of the car-body, the directions are defined as follows: $\mathrm{X}$ - longitudinal, it is from two corbels' symmetric center to headwall; Y-horizontal, it is from the symmetry of longitudinal section to sidewall; Z-vertical, it is from the brace edge beam to the roof. The finite element model is simulated with shell elements; the traction transformer is viewed as a block hanging under the car-body, where the elastic spring unit (COMBIN14) is used to connect the car-body and traction transformer [5]. Car-body-traction transformer simulation model is shown in Fig.3.

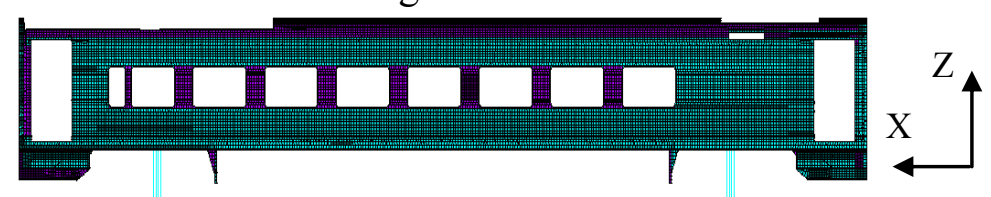

(a) Car-body model

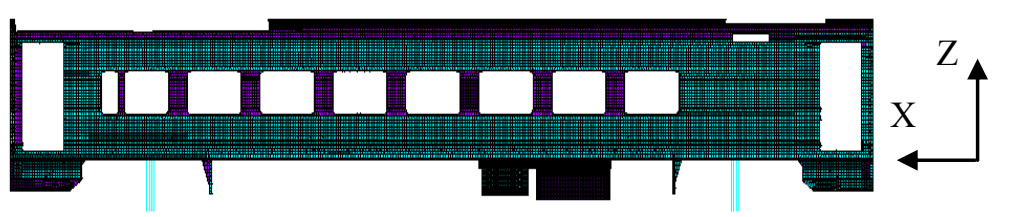

(b) Car-body-traction transformer model

Fig. 3 Simulation Models

\section{The Transformer's Effect on the Natural Frequency of the System}

Take vertical bending mode for example [6].

When the vehicle is not hanging transformer, the car-body vertical bending mode frequency is 17.46Hz, as shown in Fig.4. 


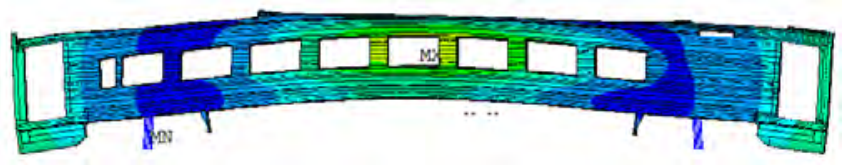

Fig. 4 Car-body Vertical Bending Mode (without transformer)

The result of harmonic response is shown in Fig.5. One of the peak frequencies is $17.46 \mathrm{~Hz}$, corresponding car-body vertical bending mode.

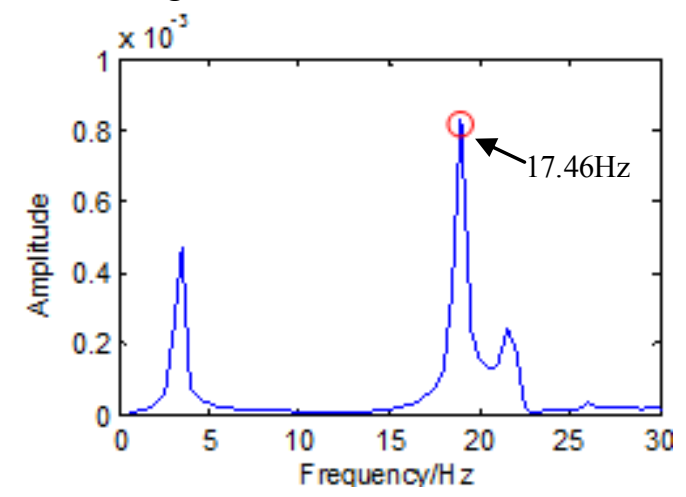

Fig. 5 Harmonic Response Results (without transformer)

When the traction transformer is hung, traction transformer's plunging mode frequency is $2.32 \mathrm{~Hz}$, shown in Fig.6.

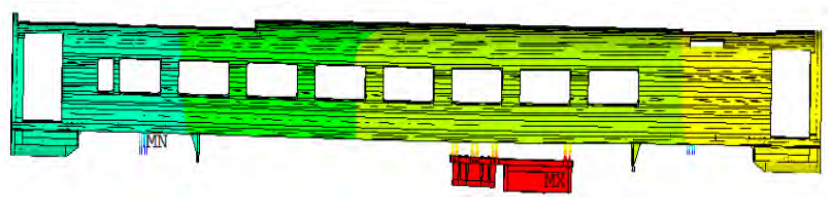

Fig. 6 Transformer Plunging Mode

The result of harmonic response is shown in Fig.7.

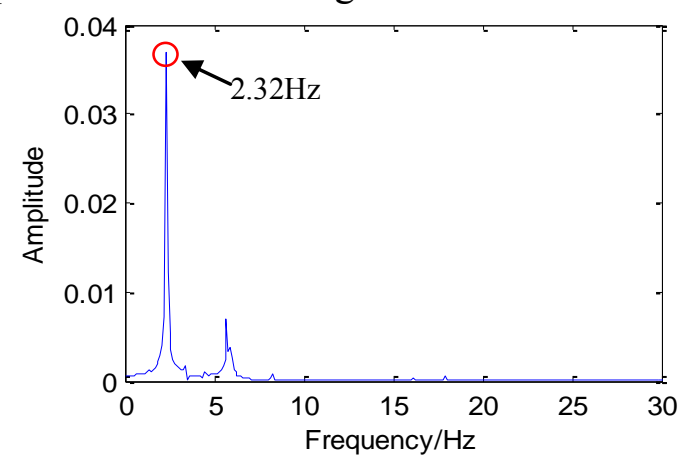

Fig. 7 Harmonic Response Results of Traction Transformer

The car-body vertical bending mode frequency is $17.89 \mathrm{~Hz}$, as shown in Fig. 8 .

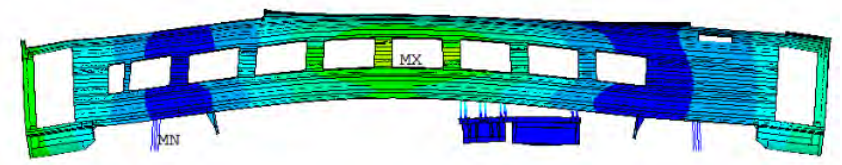

Fig. 8 Car-body Vertical Bending Mode (with transformer)

The result of harmonic response is shown in Fig.9, where $2.32 \mathrm{~Hz}$ is traction transformer plunging 
mode frequency, $17.89 \mathrm{~Hz}$ is car-body vertical bending modal frequency.

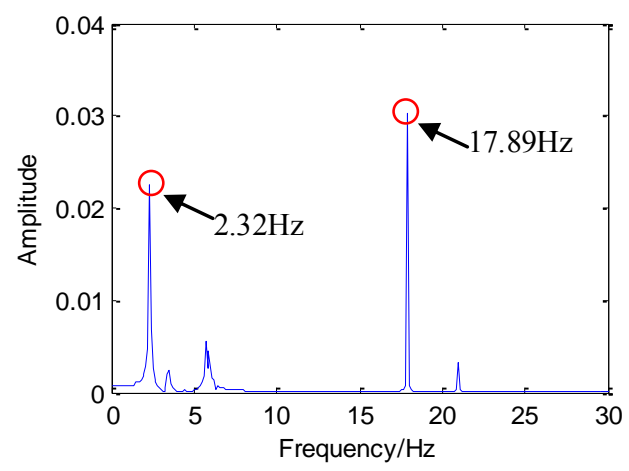

Fig. 9 Harmonic Response Results (with transformer)

Thus, when the traction transformer is hung under the car-body, the natural frequency of the new system will increase than that of original system. Hanging related devices at appropriate positions under the car-body can make the natural frequency of vehicle differ from excitation frequency. It can get the purpose of avoiding resonance.

\section{Hanging Stiffness' Effect on the Natural Frequency of System}

From Section 1, when the traction transformer is hung under the car, the following formula indicates the relationship between hanging stiffness and high natural frequency of the system:

$$
\omega_{1}=\frac{\pi^{2}}{L^{2}} \sqrt{\frac{E I}{M}+\frac{k_{1}}{M}}
$$

The derivation of $\omega_{1}$ is:

$$
\dot{\omega}_{1}=\frac{\pi^{2}}{2 L^{2} \sqrt{M\left(E I+k_{1}\right)}}>0
$$

It means that increasing hanging stiffness results in the natural frequency increasing.

To verify the relationship between hanging stiffness and the natural frequency of the system, the harmonic response calculation conditions are set up in Table 1.

Tab. 1 Harmonic Response Calculation Conditions

\begin{tabular}{ccc}
\hline Condition & Stiffness Parameter & Hanging Stiffness \\
\hline 1 & 0.5 times of the original stiffness & $15555.5 \mathrm{~N} / \mathrm{m}$ \\
2 & The original stiffness & $31111 \mathrm{~N} / \mathrm{m}$ \\
3 & 1.5 times of the original stiffness & $46666 \mathrm{~N} / \mathrm{m}$ \\
\hline
\end{tabular}

After harmonic response calculation, the vertical bending mode frequencies of car-body- traction transformer system in various conditions are shown in Table 2.

Tab. 2 Vertical Bending Mode Frequencies [Hz]

\begin{tabular}{cc}
\hline Condition & Frequency \\
\hline 1 & 17.68 \\
2 & 17.89 \\
3 & 18.10 \\
\hline
\end{tabular}

The trend is shown in Fig.10. 


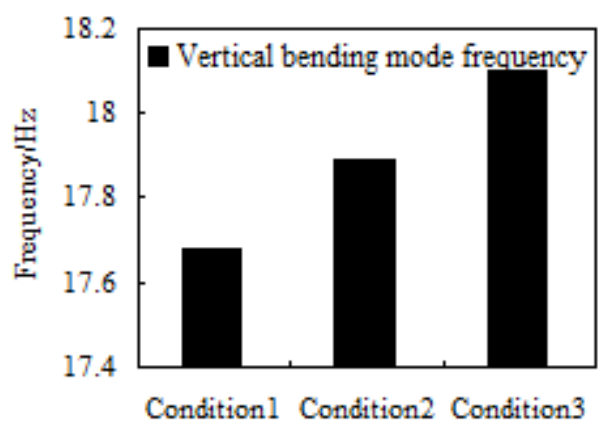

Fig. 10 Vertical Bending Mode Frequencies in Various Conditions

It can be obtained that the vertical bending mode frequency increases with increasing stiffness from Table 2. Simulation results keep pace with theory.

\section{Summary}

By theoretical analysis and simulation, the following conclusions can be got:

(1) The natural frequency of the vehicle system will increase by hanging the traction transformer.

(2) When the traction transformer hanging stiffness increases, the natural frequency of the system will increase.

\section{References}

[1]Hong-wen LIN, Mechanics of Materials fourth ed, M. Higher Education Press, Beijing, China, May 2010.

[2]Shi-jian ZHU, Jing-jun LOU, Qi-wei HE etc. Vibration Theory and Vibration Isolation, M. National Defense Industry Press, Beijing, China, June 2006, pp.52-58.

[3]Gang HAO, Zhi-cheng GUO, Li-min ZHANG. Vibration Analysis of the Suspended Equipment Coupled with Carbody, J. Railway Locomotive \& Car, Beijing, China, 2013 33(01),pp.63-64.

[4]Qiang FU, Zheng-ting GUO, The Structural Rigidity Influences on Bridge Natural Frequency and Modality, J. Shanxi Architecture, Shanxi China, 2010 36(21).

[5]Jian-hong LAN, The Stiffness Finite Element Analysis of CRH3 EMU Body, D. Chengdu, China, 2012.

[6]Hui-chao WU, Ping-bo WU, Effect of Equipment Suspension Stiffness on Riding Quality, J. Noise and Vibration Control, Shanghai, China, 2012(4).

[7]Jian-yong YU, Li-min ZHANG, Xiao-yu HUANG etc. The Influence of the Hanging Device on Complete Car's Model, J. Noise and Vibration Control, Shanghai, China 2012(05).

[8]Guang-wu YANG, Shou-ne XIAO, Analysis of Effect of Equipment with Flexible Suspension on Modal of Train Car-body in the Preparation Conditions, J. Railway Locomotive \& Car, Beijing, China,2012 32(04).

[9]Shu-ying GAO, Huo-ming SHEN, Vibration Mechanics, M. Railroad Publication House of China, Beijing, China, 2011. 